\title{
Analyticity Properties of the Weakly Coupled Double-Well Model
}

K. Klöckner and B. Ströter

Institut für Angewandte Mathematik, Johann Wolfgang Goethe-Universität, D-6000 Frankfurt am Main, Federal Republic of Germany

Abstract. In this note we study lattice $\Phi^{4}$-models with Hamiltonian

$$
H=\frac{1}{2}(\varphi,-\Delta \varphi)+\lambda \Sigma\left(\varphi_{i}^{2}-\frac{m^{2}}{8 \lambda}\right)^{2}
$$

and Gaussian boundary conditions. Using the polymer expansion we obtain analyticity of the pressure and the correlation functions in the infinite volume limit in a region

$$
\left\{\lambda|| \lambda|<\varepsilon,| \arg \lambda \mid<\frac{\pi}{2}-\delta\right\}
$$

for every $\delta>0$.

\section{Introduction}

Let us consider the $P(\varphi)$-theory on the $v$-dimensional lattice $\mathbb{Z}^{v}$ with partition function in the finite volume $\Lambda$ given by

$$
\begin{aligned}
& Z_{\Lambda}(\lambda, m)= \\
& \quad \int_{\mathbb{R}^{|\lambda|}} \exp \left(-\frac{1}{2}\left(\varphi,-\Delta_{\Lambda} \varphi\right)-\sum_{i \in \Lambda} P\left(\varphi_{i}\right)-\frac{m^{2}}{2} \sum_{i \in \partial+\Lambda}\left(\varphi_{i}-\frac{m}{\sqrt{8 \lambda}}\right)^{2}\right) \prod_{i \in \bar{\Lambda}} d \varphi_{i},
\end{aligned}
$$

where

$$
\partial_{+} \Lambda=\left\{i \in \mathbb{Z}^{\nu} \backslash \Lambda|\exists j \in \Lambda, \quad| i-j \mid=1\right\}
$$

and

$$
\bar{\Lambda}=\Lambda \cup \partial_{+} \Lambda .
$$

We study the case

$$
P(\varphi)=\lambda\left(\varphi^{2}-\frac{m^{2}}{8 \lambda}\right)^{2},
$$


which is a polynomial with two minima $\pm \xi, \xi=m / \sqrt{8 \lambda} . \Delta_{\Lambda}$ is the lattice Laplace operator with zero Dirichlet boundary conditions on $\partial_{+} \Lambda$ which decouples $\bar{A}$ from its exterior. The third term in the exponent defines positive Gaussian boundary conditions.

Equation (1.1) can be interpreted as a continuous-spin Ising model with two phases, which are well separated if $\lambda$ is sufficiently small and $m$ large. For real $\lambda$, it was shown by Constantinescu and Ströter [3], that the infinite volume limits of pressure and correlation functions exist for such $\lambda$ and that a modified model with interaction $v^{-1}\left(\lambda q^{4}-\left(m^{2} / 4\right) q^{2}\right)$ converges to the Ising-model for $v \rightarrow 0$.

It has long been an open question whether this model is Borel summable in the coupling constant $\lambda$ for $\lambda \rightarrow 0$. In order to prove or disprove this it is necessary to study analyticity properties. For $P(\varphi)$-models with one minimum this has been done by Constantinescu and Klöckner [2].

\section{Discussion of the Main Results}

In order to show Borel summability, one has to prove analyticity in a domain $\{\lambda,|\lambda|<R$ and $|\arg \lambda|<\pi / 2\}$ with uniform factorial bounds on the asymptotic expansion, see Nevanlinna [6], or alternatively in a domain $\left\{\lambda, \operatorname{Re} \lambda^{-1}>R^{-1}\right\}$, see Sokal [9]. We do not quite obtain these results. In fact, if we study a sector with opening angle $\theta$, the radius of analyticity seems to shrink with $\cos \theta$. This could be an indication that Borel summability does not hold.

We now state our main result:

Theorem 1. Consider the $P(\varphi)$-model on $\mathbb{Z}^{v}$ with finite volume partition function given by (1.l). Let $A$ be an arbitrary multi-index on $\mathbb{Z}^{v}$ with $A(\varphi)=\prod_{i \in \operatorname{supp} A} \varphi_{i}^{A(i)}$ and

$$
\begin{aligned}
&\langle A\rangle_{\Lambda}(\lambda, m) \\
&=Z_{\Lambda}^{-1}(\lambda, m) \int_{\mathbb{R}|\bar{\lambda}|} A(\varphi) \exp \left(-\frac{1}{2}\left(\varphi,-\Delta_{\Lambda} \varphi\right)-\sum_{i \in \Lambda} P\left(\varphi_{i}\right)\right) \\
& \cdot \exp \left(-\frac{m^{2}}{2} \sum_{i \in \partial+\Lambda}\left(\varphi_{i}-\xi\right)^{2}\right) \prod_{i \in \bar{\Lambda}} d \varphi_{i} .
\end{aligned}
$$

Let

$$
P_{\Lambda}(\lambda, m)=|\Lambda|^{-1} \log Z_{\Lambda}^{-1}(\lambda, m) .
$$

Then for every $\delta>0$ there exists an $\varepsilon>0$, such that for $m$ large and $\lambda \in\{\lambda \|$ arg $\lambda \mid \leqq$ $\pi / 2-\delta,|\lambda|<\varepsilon\}$ the infinite volume limits of $\langle A\rangle_{A}$ and $P_{A}$ exist and are analytic.

The proof of this theorem closely follows the spirit of mean field theory developed by Glimm, Jaffe and Spencer [5], see also Constantinescu and Ströter [3]. The well-known contour-cluster expansion is rewritten in terms of polymers (cf. Bricmont, Lebowitz and Pfister [1]), which is probably the most sophisticated algebraic formalism for studying the infinite volume limit of the pressure. Adapting a trick by E. Seiler [8], we then obtain the result for correlation functions. 
Applying the algebraic formalism the proof reduces to showing the exponential decay of the Boltzmann factors of the polymers. We have to estimate the behaviour of certain one-dimensional integrals using the complex Laplace method. Here we fail to extend our results to a larger domain of analyticity. Our estimates are uniform in $\Lambda$. Combining this with pointwise convergence for real $\lambda[3]$ we can apply Vitali's theorem to obtain Theorem 1.

\section{The Expansion}

In this part we give the expansion in polymers which yields our results. It has two main ingredients: an expansion in phase boundaries and a cluster expansion in the regions of pure phase.

First we map our field variable $\varphi_{i}$ onto Ising spin variables $\sigma_{i}$. Let $\sigma_{i} \in\{+1,-1\}$. Here $\Sigma \equiv\left\{\sigma_{i}\right\}_{i \in \Lambda} \in\{+1,-1\}^{\Lambda}$ is called a (spin) configuration. Setting

$$
\chi_{+}(\varphi)=\pi^{-1 / 2} \int_{0}^{\infty} \exp \left(-(\varphi-z)^{2}\right) d z=\chi_{-}(-\varphi)
$$

we have a partition of unity with $\chi_{+}(\varphi)+\chi_{-}(\varphi)=1$. To each configuration $\Sigma$ we attribute

Thus we have

$$
\chi_{\Sigma}(\varphi)=\prod_{i \in \Lambda} \chi_{\sigma_{2}}\left(\varphi_{i}\right)
$$

$$
Z_{\Lambda}(\lambda, m)=\sum_{\Sigma \in\{ \pm 1\}^{A}} Z_{A, \Sigma}(\lambda, m)
$$

where

$$
\begin{aligned}
Z_{\Lambda, \Sigma}(\lambda, m)=\int_{\mathbb{R} \mid \overline{\mid \overline{\mid}}} \chi_{\Sigma}(\varphi) \exp \left\{-\frac{1}{2}\left(\varphi,-\Delta_{\Lambda} \varphi\right)-\sum_{i \in \Lambda} P\left(\varphi_{i}\right)\right. \\
\left.-\frac{m^{2}}{2} \sum_{i \in \hat{\partial}+\Lambda}\left(\varphi_{i}-\xi\right)^{2}\right\} \prod_{i \in \bar{\Lambda}} d \varphi_{i} .
\end{aligned}
$$

Seeing that the partition function has positive Gaussian boundary conditions we can set $\sigma_{i}=1$ for $i \in \partial_{+} \Lambda$ and extend the configuration to $\bar{\Lambda}$ (without changing $\chi_{\Sigma}$ ).

If $\Sigma$ is a configuration, let

$$
\Sigma^{*}=\left\{i \in \bar{\Lambda} \mid \exists j \in \bar{\Lambda} \quad \sigma_{i} \sigma_{j}=-1 \text { and }|i-j|=1\right\}
$$

denote the set of phase boundaries. We can identify $\Sigma$ with the set of bonds $(i j)$ with $\sigma_{i} \sigma_{j}=-1$ (or with the bonds in the dual lattice perpendicular to $(i j)$ ). Here $\Sigma^{c}$ is its complement in $\bar{\Lambda}$, i.e. the set of bonds $(i j)$ with $\sigma_{i} \sigma_{j}=1$ and $|\Sigma|$ and $\left|\Sigma^{c}\right|$ are the cardinalities of $\Sigma, \Sigma^{c}$ respectively.

For each $i \in \Lambda$ we set

$$
b_{i}=b_{i}(\Sigma)=\mid\left\{j \in \Lambda|| i-j \mid=1 \text { and } \sigma_{i} \sigma_{j}=-1\right\} \mid .
$$

We have $b_{i} \leqq 2 v$ and $b_{i}=0$ unless $i \in \Sigma^{*}$. Furthermore set

$$
a_{i}=\left|\left\{j \in \mathbb{Z}^{v},|i-j|=1, j \in \Lambda\right\}\right|, e_{i}=\frac{1}{2}\left(2 v-a_{i}\right) .
$$

We find $a_{i}<2 v$ for $i \in \partial_{+} \Lambda$. 
For each configuration $\Sigma=\left\{\sigma_{i}\right\}_{i \in \bar{\Lambda}}$ we can now translate the coordinates $\varphi_{i}$ into the corresponding minima $h_{i}=\sigma_{i} \xi$. Let the translated field be denoted by

$$
\psi_{i}=\varphi_{i}-h_{i}
$$

We now express the Hamiltonian in terms of the translated variables. Some straightforward calculations yield

$$
\begin{gathered}
\frac{1}{2}\left(\varphi,-\Delta_{\Lambda} \varphi\right)=\frac{1}{2}\left(\psi,-\Delta_{\Lambda} \psi\right)+\xi^{2}\left(v|\bar{\Lambda}|+|\Sigma|-\left|\Sigma^{c}\right|\right) \\
+2 \xi \sum_{i \in \bar{\Lambda}} c_{i} \sigma_{i} \psi_{i},
\end{gathered}
$$

where

$$
c_{i}=\left\{\begin{array}{l}
b_{i}, \quad i \in \Lambda \\
b_{i}+e_{i}, \quad i \in \partial_{+} \Lambda .
\end{array}\right.
$$

Note that $\left(v|\Lambda|+|\Sigma|-\left|\Sigma^{c}\right|\right)=\sum_{i \in \Lambda} c_{i}=\sum_{i \in \Sigma^{*} \cup \partial_{+} \Lambda} c_{i}$, because $c_{i}>0$ for $i \in \Sigma^{*} \cup \partial_{+} \Lambda$ and $c_{i}=0$ for $i \in \Lambda \backslash \Sigma^{*}$. For the interaction we get

$$
P\left(\varphi_{i}\right)=\lambda \psi_{i}^{4}+\sigma_{i} m \sqrt{2 \lambda} \psi_{i}^{3}+\frac{m^{2}}{2} \psi_{i}^{2} .
$$

Scaling with $\sigma_{i}$ we obtain

$$
Q\left(\psi_{i}\right)=\lambda \psi_{i}^{4}+m \sqrt{2 \lambda} \psi_{i}^{3}+\frac{m^{2}}{2} \psi_{i}^{2}
$$

The partition of unity is transformed in the following way

$$
\begin{aligned}
\chi_{\sigma_{t}}\left(\varphi_{i}\right) & =\chi_{\sigma_{i}}\left(\psi_{i}+\sigma_{i} \xi\right), \\
\chi_{\sigma_{t}}\left(\sigma_{i} \psi_{i}+\sigma_{i} \xi\right) & =\chi_{\sigma_{t}}\left(\sigma_{i}\left(\psi_{i}+\xi\right)\right)=\chi_{+}\left(\psi_{i}+\xi\right) .
\end{aligned}
$$

Inserting (3.2) in (3.1b) and scaling with $\sigma_{i}$ we arrive at

$$
\begin{gathered}
Z_{\Lambda, \Sigma}(\lambda, m)=(-1)^{|\Lambda-(\Sigma)|} \exp \left(-\xi^{2}\left(v|\bar{\Lambda}|+|\Sigma|-\left|\Sigma^{c}\right|\right)\right) \\
\int_{\mathbb{R}|\bar{\Lambda}|} \prod_{i \in \Lambda} \chi_{+}\left(\psi_{i}+\xi\right) \exp \left(\sum_{|i-j|=1} \sigma_{i} \sigma_{j} \psi_{i} \psi_{j}-\sum_{i \in \Sigma^{*} \cup \partial_{+} \Lambda} 2 c_{i} \xi \psi_{i}\right) \\
\cdot \exp \left(-\sum_{i \in \Lambda} Q\left(\psi_{i}\right)-\frac{1}{2} m^{2} \sum_{i \in \partial+\Lambda} \psi_{i}^{2}-v \sum_{i \in \Lambda} \psi_{i}^{2}\right) \prod_{i \in \Lambda} d \psi_{i} .
\end{gathered}
$$

Here $\Lambda_{-}(\Sigma)=\left|\left\{i \in \Lambda, \sigma_{i}=-1\right\}\right|$.

Having expanded in phase boundaries or "contours" we now turn to the pure phases. We study the term (3.3) coupling next-neighbour spins and apply the Mayer trick:

$$
\begin{gathered}
\exp \left(\sum_{|i-j|=1} \sigma_{i} \sigma_{j} \psi_{i} \psi_{j}\right)=\prod_{(i j) \in \Sigma} \exp \left(-\psi_{i} \psi_{j}\right) \prod_{(i j) \in \Sigma^{c}} \exp \left(\psi_{i} \psi_{j}\right), \\
\prod_{(i j) \in \Sigma^{c}} \exp \left(\psi_{i} \psi_{j}\right)=\sum_{X \in \Sigma^{c}} \prod_{(i j) \in X}\left(\exp \left(\psi_{i} \psi_{j}\right)-1\right) .
\end{gathered}
$$


Here $X$ is a set of bonds. The corresponding set of points belonging to bonds in $X$ is denoted by $X^{*}$. Inserting this in (3.1) and resumming we obtain

$$
\begin{aligned}
Z_{\Lambda}(\lambda, m)= & \sum_{\Sigma}(-1)^{|\Lambda-(\Sigma)|} \exp \left(-\xi^{2}\left(v|\bar{\Lambda}|+|\Sigma|-\left|\Sigma^{c}\right|\right)\right) \\
& \cdot \sum_{X \subset \Sigma^{c}} Z_{\Lambda, \Sigma, X}(\lambda, m) \prod_{i \notin\left(\Sigma^{*} \cup X^{*}\right)} g_{i}(\lambda, m),
\end{aligned}
$$

where

and

$$
\begin{aligned}
Z_{\Lambda, \Sigma, X}(\lambda, m)= & \int_{\mathbb{R}^{\left|\Sigma^{*} \cup X^{*}\right|}} \prod_{(i j) \in \Sigma} \exp \left(-\psi_{i} \psi_{j}\right) \prod_{(i j) \in X}\left(\exp \left(\psi_{i} \psi_{j}\right)-1\right) \\
& \cdot \prod_{i \in\left(\Sigma^{*} \cup\left(\partial+\Lambda \cap X^{*}\right)\right.} \exp \left(-2 c_{i} \xi\right) \prod_{i \in \partial+\Lambda \cap\left(\Sigma^{*} \cup X^{*}\right)} \exp \left(-\frac{1}{2} m^{2} \psi_{i}^{2}\right) \\
& \cdot \prod_{i \in \Lambda \cap\left(\Sigma^{*} \cup X^{*}\right)} \chi_{+}\left(\psi_{i}+\xi\right) \exp \left(-Q\left(\psi_{i}\right)\right) \prod_{i \in \Sigma^{*} \cup X^{*}} e^{-v \psi_{i}^{2}} d \psi_{i}
\end{aligned}
$$

$$
g_{i}(\lambda, m)=\left\{\begin{array}{l}
\int_{-\infty}^{\infty} \chi_{+}(\varphi) \exp \left(-P(\varphi)-v(\varphi-\xi)^{2}\right) d \varphi, \quad i \in \Lambda \\
\exp \left(-\xi^{2} e_{i}\right) \int_{-\infty}^{\infty} \exp \left(-\frac{1}{2}\left(m^{2}+2 v\right) \psi_{i}^{2}-2 e_{i} \xi \psi_{i}\right) d \psi_{i}, \quad i \in \partial_{+} \Lambda
\end{array}\right.
$$

The properties of the $g_{i}(\lambda, m)$ for small $\lambda$ are essential in proving convergence of our contour-cluster expansion (3.4a). We will briefly summarize the results, the proofs can be found in the appendix.

Completing the square we can easily evaluate the integral for $i \in \partial_{+} \Lambda$ :

$$
g_{i}(\lambda, m)=\sqrt{2 \pi}\left(m^{2}+2 v\right)^{-1 / 2} \exp \left(\frac{1}{2} e_{i} m^{2}\left(m^{2}+2 v\right)^{-1} \lambda^{-1}\right) \exp \left(-\xi^{2} e_{i}\right) .
$$

For $i \in \Lambda$ we gel

$$
\begin{aligned}
\left|g_{i}(\lambda, m)\right| \geqq|\tilde{g}(\lambda, m)| & \sim\left|\int_{0}^{\infty} \exp \left(-P(\varphi)-v(\varphi-\xi)^{2}\right) d \varphi\right| \\
& \sim 2 \pi^{1 / 2}\left(m^{2}-2 v\right)^{-1 / 2}
\end{aligned}
$$

uniformly in any sector $|\arg \lambda| \leqq \pi / 2-\delta$ for $|\lambda| \rightarrow 0$. This gives us $g_{i}(\lambda, m) \neq 0$ for $|\lambda|$ small and $|\arg | \leqq \pi / 2-\delta$.

We can now map our expansion onto a "polymer system." Let $\Gamma=(\Sigma \cup X)$, where $\Gamma$ is called connected if any two points in $\Sigma^{*} \cup X^{*}$ can be connected by a path in $\Sigma \cup X$. Thus $\Gamma$ can be decomposed into connected components and we notice that

a) the sum (3.4a) runs over all admissible (i.e. pairwise disconnected) families of connected $\Gamma$,

b) $Z_{\Lambda, \Sigma, X}(\lambda, m)$ factorizes over the connected components of $\Gamma=(\Sigma, X)$. The connected components of $\Gamma$ are called polymers $p$, and $\Gamma=\left(p_{1}, \ldots, p_{n}\right)$.

Let

$$
\begin{aligned}
\tilde{\phi}_{\lambda, m}(\Sigma, X)= & (-1)^{|\Lambda-(\Sigma)|} \exp \left(-\xi^{2}\left(v\left|\bar{\Lambda} \cap \Gamma^{*}\right|+|\Sigma|-\left|\Sigma^{c} \cap \Gamma\right|\right) Z_{\Lambda, \Sigma, X}(\lambda, m)\right. \\
& \cdot\left(\prod_{i \in\left(\Sigma^{*} \cup X^{*}\right)} g_{i}(\lambda, m)\right)^{-1}
\end{aligned}
$$




$$
\begin{aligned}
\tilde{Z}_{\Lambda}(\lambda, m) & =\left(\prod_{i \in \Lambda} g_{i}(\lambda, m)\right)^{-1} Z_{\Lambda}(\lambda, m)=\sum_{\Gamma=(\Sigma, X)} \tilde{\phi}_{\lambda, m}(\Sigma, X) \\
& =\sum_{\Gamma=\left(p_{1}, \ldots, p_{n} \Gamma\right)} \prod_{i=1}^{n \Gamma} \tilde{\phi}_{\lambda, m}\left(p_{i}\right) .
\end{aligned}
$$

In the next chapter we will prove the basic estimate which controls the "Boltzmann factors" $\tilde{\phi}_{\lambda, m}(p)$ for polymers $p$.

\section{Estimates for $\tilde{\phi}_{\lambda, m}\left(p_{i}\right)$}

For $\Gamma=(\Sigma, X)$, define $|\Gamma|=\left|\Sigma^{*} \cup X^{*}\right|$ and $|p|$ accordingly. We will prove

Lemma 1. Let $\Gamma=(\Sigma, X)$ as in Sect. 3. For every $\delta>0$ there exist $\varepsilon>0$ and $k>0$, such that for $m$ large and $\lambda \in\{\lambda|| \arg \lambda|\leqq \pi / 2-\delta,| \lambda \mid<\varepsilon\}$ we have the following estimate

$$
\left|\widetilde{\phi}_{\lambda, m}(\Gamma)\right| \leqq \exp (-k|\Gamma|) .
$$

The proof is closely related to the methods for proving convergence of the cluster expansion in Glimm-Jaffe-Spencer [5] and Constantinescu-Ströter [3] and uses Hölder's inequality to separate the terms coming from the contour expansion from those resulting from the Mayer trick.

Let (ij) be a bond. We have

$$
\exp \left(\psi_{i} \psi_{j}\right)-1=\int_{0}^{1} \psi_{i} \psi_{j} \exp \left(\alpha_{i j} \psi_{i} \psi_{j}\right) d \alpha_{i j} .
$$

Setting $d_{i}=\left|\left\{j \in X^{*},|i-j|=1\right\}\right|, d \alpha=\prod_{(i j) \in X} d \alpha_{i j}$ and $p=(\Sigma, X), p^{*}=\Sigma^{*} \cup X^{*}$, we obtain from (3.4b)

$$
\begin{aligned}
Z_{\Lambda, \Sigma, X}(\lambda, m)= & \int_{0}^{1} \int_{\mathbb{R}^{\left|p^{*}\right|}}\left\{\prod_{i \in X^{*}} \psi_{i}^{d_{i}} \prod_{(i j) \in \Sigma} \exp \left(-\psi_{i} \psi_{j}\right) \prod_{(i j) \in X} \exp \left(\alpha_{i j} \psi_{i} \psi_{j}\right)\right. \\
& \cdot \prod_{i \in \Sigma^{*} \cup\left(\partial_{+} \Lambda \cap X^{*}\right)} \exp \left(-2 c_{i} \xi \psi_{i}\right) \prod_{i \in \partial+\Lambda \cap p^{*}} \exp \left(-\frac{1}{2} m^{2} \psi_{i}^{2}\right) \\
& \left.\cdot \prod_{i \in \Lambda \cap p^{*}} \chi_{+}\left(\psi_{i}+\xi\right) \exp \left(-Q\left(\psi_{i}\right)\right) \prod_{i \in p^{*}} \exp \left(-v \psi_{i}^{2}\right) d \psi_{i}\right\} d \alpha .
\end{aligned}
$$

We will now find an upper bound for $Z_{\Lambda, \Sigma, X}(\lambda, m)$ by scaling $\psi$ with $\sqrt{\lambda}$ and replacing $\lambda$ by $\lambda^{\prime}=|\lambda|(\cos \theta)^{-1}$, where $\theta=\arg \lambda$.

Note that

$$
\left|\chi_{+}\left(\frac{\psi_{i}}{\sqrt{\lambda}}+\xi\right)\right| \leqq(\cos \theta)^{-1 / 2} \chi_{+}\left(\frac{\psi_{i}}{\sqrt{\lambda^{\prime}}}+\xi^{\prime}\right)
$$

where we have put $\xi^{\prime}=m\left(8 \lambda^{\prime}\right)^{-1 / 2}$. We find after some lengthy but straight-forward calculations, involving a rescaling with $\lambda^{\prime-1 / 2}$

$$
\left|Z_{\Lambda, \Sigma, X}(\lambda, m)\right| \leqq \cos \theta^{-\left(\left|p^{*}\right|+(1 / 2) \Sigma เ \in x^{*} d_{1}\right)} \hat{Z}_{\Lambda, \Sigma, X}\left(\lambda^{\prime}, m\right)
$$


where

$$
\begin{aligned}
& \hat{Z}_{\Lambda, \Sigma, X}\left(\lambda^{\prime}, m\right)=\int_{0}^{1} \int_{\mathbb{R}^{\left|p^{*}\right|}}\left\{\prod_{i \in X^{*}}\left|\psi_{i}\right|^{d_{i}} \prod_{(i j) \in \Sigma} \exp \left(-\psi_{i} \psi_{j}\right) \prod_{(i j) \in X} \exp \left(\alpha_{i j} \psi_{i} \psi_{j}\right)\right. \\
& \cdot \prod_{i \in \Sigma^{*} \cup\left(\partial+\Lambda \cap X^{*}\right)} \exp \left(-2 c_{i} \xi^{\prime} \psi_{i}\right) \prod_{i \in \partial+\Lambda \cap p^{*}} \exp \left(-\frac{1}{2} m^{2} \psi_{1}^{2}\right) \\
& \left.\cdot \prod_{i \in \Lambda \cap p^{*}} \chi_{+}\left(\psi_{i}+\xi^{\prime}\right) \exp \left(-\left(\lambda^{\prime} \psi_{i}^{4}+m \sqrt{2 \lambda^{\prime}} \psi_{i}^{3}+\frac{m^{2}}{2} \psi_{i}^{2}\right)\right)\right\} \\
& \cdot \prod_{i \in p^{*}} \exp \left(-v \psi_{i}^{2}\right) d \psi_{i} d \alpha .
\end{aligned}
$$

We see that the quality of the estimate depends on the sector which we study.

We will now apply Hölder's inequality to (4.2) with respect to the measures $\exp \left(-v \psi_{i}^{2}\right) d \psi_{i}, d \alpha$

$$
\begin{aligned}
& \left|Z_{\Lambda, \Sigma, X}(\lambda, m)\right|^{2} \leqq(\cos \theta)^{-2\left|p^{*}\right|-\Sigma_{i \in X} d_{i}} \\
& \quad \cdot \int_{0}^{1} \int_{\mathbb{R}^{\left|p^{*}\right|}}\left\{\prod_{i \in X^{*}}\left|\psi_{i}\right|^{2 d_{i}} \prod_{(i j) \in X} \exp \left(2 \alpha_{i j} \psi_{i} \psi_{j}\right)\right. \\
& \cdot \prod_{i \in \Lambda \cap p^{*}} \chi_{+}\left(\psi_{i}+\xi^{\prime}\right)^{2} \exp \left(-2 \lambda^{\prime} \psi_{i}^{4}-2 m \sqrt{2 \lambda^{\prime}} \psi_{i}^{3}\right) \\
& \left.\cdot \prod_{i \in p^{*}} \exp \left(-\left(\eta m^{2}+v\right) \psi_{i}^{2}\right) d \psi_{i} d \alpha\right\} \int_{\mathbb{B}^{\left|p^{*}\right|}} \prod_{(i j) \in \Sigma} \exp \left(-2 \psi_{i} \psi_{j}\right) \\
& \quad \cdot \prod_{i \in \Sigma^{*} \cup\left(\partial+\Lambda \cap X^{*}\right)} \exp \left(-4 c_{i} \xi^{\prime} \psi_{i}\right) \prod_{i \in p^{*}} \exp \left(-\left((1-\eta) m^{2}+v\right) \psi_{i}^{2}\right) d \psi_{i} .
\end{aligned}
$$

We have chosen $\eta \in(0,1)$ and $m^{2}$ large, such that both $(1-\eta) m^{2}$ and $\eta m^{2}$ are greater than $v$.

We can now estimate the factors separately. We will first treat the second integral in (4.3) which comprises the phase boundary terms.

a) Phase Boundary Terms

This integral can be majorized by

$$
\begin{aligned}
& \left(\pi^{1 / 2}\left((1-\eta) m^{2}+v\right)^{-1 / 2}\right)^{\left|X^{*} \backslash\left(\Sigma^{*} \cup \partial+\Lambda\right)\right|} \\
& \cdot \prod_{i \in\left(X^{*} \cap \partial+\Lambda\right) \backslash \Sigma^{*}} \int_{-\infty}^{\infty} \exp \left(-4 c_{i} \xi^{\prime} \psi_{i}-\left((1-\eta) m^{2}+v\right) \psi_{i}^{2}\right) d \psi_{i} \\
& \cdot \int_{\mathbb{R}^{\left|\Sigma^{*}\right|}} \prod_{(i j) \in \Sigma} \exp \left(\psi_{i}^{2}+\psi_{j}^{2}\right) \prod_{i \in \Sigma^{*}} \exp \left(-4 c_{i} \xi^{\prime} \psi_{i}\right) \exp \left(-\left((1-\eta) m^{2}+v\right) \psi_{i}^{2}\right) \mathrm{d} \psi_{i} .
\end{aligned}
$$

The second and third factor can be estimated by completing the square so that we can majorize the integral by

$$
\begin{aligned}
& \pi^{1 / 2\left|p^{*}\right|}\left((1-\eta) m^{2}+v\right)^{-1 / 2\left|X^{*}\right| \Sigma^{*} \mid}\left((1-\eta) m^{2}-v\right)^{-1 / 2\left|\Sigma^{*}\right|} \\
& \quad \cdot \prod_{i \in \Sigma^{*}} \exp \left(4 c_{i}^{2} \xi^{\prime 2}\left((1-\eta) m^{2}-v\right)^{-1}\right) \prod_{\Lambda i \in\left(X^{*} \cap \hat{\theta}+\Lambda\right)} \exp \left(4 c_{i}^{2} \xi^{\prime 2}\left((1-\eta) m^{2}+v\right)^{-1}\right) .
\end{aligned}
$$


In the final estimate of $\phi_{\lambda, m}(\Sigma, X)$ the exponential divergencies are outweighed by $\exp \left(-\xi^{2}\left(v|\Lambda|+|\Sigma|-\left|\Sigma^{c}\right|\right)\right)$ which also comes from (3.2a).

\section{b) Pure Phase Regions}

We will now study the first integral in (4.3) comprising terms which result from the Mayer trick. Using $2 \alpha_{i j} \psi_{i} \psi_{j} \leqq \psi_{i}^{2}+\psi_{j}^{2}$ we bound this factor by

$$
\begin{gathered}
\int_{\mathbb{R}^{|p *|}}\left\{\prod_{i \in X^{*}}\left|\psi_{i}\right|^{2 d_{i}} \prod_{i \in \Lambda \cap p^{*}} \chi_{+}^{2}\left(\psi_{i}+\xi^{\prime}\right) \exp \left(-2 \lambda^{\prime} \psi_{i}^{4}-2 m \sqrt{2 \lambda^{\prime}} \psi_{i}^{3}\right)\right. \\
\left.\cdot \prod_{i \in \Sigma^{*} \mid X^{*}} \exp \left(-\left(\eta m^{2}+v\right) \psi_{i}^{2}\right) \prod_{i \in X^{*}} \exp \left(-\left(\eta m^{2}-v\right) \psi_{i}^{2}\right)\right\} \prod_{i \in p^{*}} d \psi_{i} .
\end{gathered}
$$

The integral over $\mathbb{R}^{\left|p^{*}\right|}$ factorizes into one-dimensional integrals and we get the following cases:

1.) $i \in \partial_{+} \Lambda \cap X^{*}$ :

$$
\int_{-\infty}^{\infty}\left|\psi_{i}\right|^{2 d_{i}} \exp \left(-\left(\eta m^{2}-v\right) \psi_{i}^{2}\right) d \psi_{i}=\left(\eta m^{2}-v\right)^{-d_{l}-1 / 2} \Gamma\left(d_{i}+\frac{1}{2}\right) .
$$

2.) $i \in \partial_{+} \Lambda \cap\left(\Sigma^{*} \backslash X^{*}\right)$ :

$$
\int_{-\infty}^{\infty} \exp \left(-\left(\eta m^{2}+v\right) \psi_{i}^{2}\right) d \psi_{i}=\pi^{1 / 2}\left(\eta m^{2}+v\right)^{-1 / 2}
$$

3.) $i \in\left(\Sigma^{*} \backslash X^{*}\right) \cap \Lambda$ :

$$
\begin{aligned}
& \int_{-\infty}^{\infty} \chi_{+}^{2}\left(\psi_{i}+\xi^{\prime}\right) \exp \left(-2 \lambda^{\prime} \psi_{i}^{4}-2 m \sqrt{2 \lambda^{\prime}} \psi_{i}^{3}-\left(\eta m^{2}+v\right) \psi_{i}^{2}\right) d \psi_{i} \\
= & \int_{-\infty}^{\infty}\left\{\exp \left(-2\left(\lambda^{\prime}\left(\varphi^{2}-\xi^{\prime 2}\right)^{2}-(1-\eta+\varepsilon) \frac{m^{2}}{2}\left(\varphi-\xi^{\prime}\right)^{2}-\log \chi_{+}(\varphi)\right)\right)\right. \\
& \left.\cdot \exp \left(-\left(v+\varepsilon m^{2}\right)\left(\varphi-\xi^{\prime}\right)^{2}\right)\right\} d \varphi
\end{aligned}
$$

If $\varepsilon$ and $\eta$ are chosen such that $1-\eta+\varepsilon<\frac{1}{36}$ we can apply a lemma by Constantinescu and Ströter [3] which gives us $\lambda^{\prime}\left(\varphi^{2}-\xi^{\prime 2}\right)^{2}-(1-\eta+\varepsilon)\left(m^{2} / 2\right) \times$ $\left(\varphi-\xi^{\prime}\right)^{2}-\log \chi_{+}(\varphi) \geqq 0$. We can majorize our integral by

$$
\pi^{1 / 2}\left(v+\varepsilon m^{2}\right)^{-1 / 2}
$$

4.) $i \in X^{*} \cap \Lambda$ :

In this case we must choose $\varepsilon, \eta$ such that $1-\eta+\varepsilon+v / m^{2}<\frac{1}{36}$, we can then apply the above lemma and obtain an upper bound

$$
\left(\varepsilon m^{2}\right)^{-d_{i}-1 / 2} \Gamma\left(d_{i}+1 / 2\right) .
$$

1.) - 4.) is basically a very simple way of estimating covariances by Gaussian integrals. 


\section{c) Final Estimates}

Lumping together all the terms from (4.4) and (4.5) and using the properties of the $g_{i}(\lambda, m)$ we obtain the final estimate for $\phi_{\lambda, m}(\Sigma, X)$. The main factor of convergence coming from the phase boundary or contour expansion is $\exp \left(-2 \xi^{\prime 2}(v \mid \Lambda)+\right.$ $\left.\left.|\Sigma|-\left|\Sigma^{c}\right|\right)\right)$ or $\exp \left(-2 \xi^{\prime 2} \sum_{i \in \Sigma^{*}} b_{i}\right)$. From the cluster expansion we derive convergence factors

$$
\left(\delta m^{2}\right)^{-1 / 2+} d_{i}, \quad \delta>0, \text { for every } i \in X
$$

which cancel the divergence from the $g_{i}(\lambda, m)$. We have asymptotically for $\lambda \rightarrow 0$ in a sector with opening angle less than $\pi$

$$
\begin{aligned}
& \left|\tilde{\phi}_{\lambda, m}(\Sigma, X)\right|^{2} \leqq\left\{\exp \left(-2 \xi^{\prime 2} \sum_{i \in \Sigma^{*}} b_{i}\right) \prod_{i \in \Sigma^{*}} \exp \left(4 c_{i}^{2} \xi^{\prime 2}\left((1-\eta) m^{2}-v\right)^{-1}\right)\right. \\
& \left.\prod_{i \in\left(X^{*} \cap \partial+\Lambda\right)} \exp \left(4 c_{i}^{2} \xi^{\prime 2}\left((1-\eta) m^{2}+v\right)^{-1}\right) \prod_{i \in \partial+\Lambda \cap\left(\Sigma^{*} \cup X^{*}\right)} \exp \left(-e_{i}^{2} m^{2}\left(m^{2}+2 v\right)^{-1} \lambda^{\prime-1}\right)\right\} \\
& \left\{\left((1-\eta) m^{2}+v\right)^{-1 / 2\left|X^{*}\right| \Sigma^{*} \mid}\left((1-\eta) m^{2}-v\right)^{-1 / 2\left|\Sigma^{*}\right|} \prod_{i \in \partial+\Lambda \cap X^{*}}\left(\eta m^{2}-v\right)^{-d_{i}-1 / 2}\right. \\
& \quad \prod_{i \in \partial+\Lambda \cap\left(\Sigma^{*} \mid X^{*}\right)}\left(\eta m^{2}+v\right)^{-1 / 2} \prod_{i \in X^{*} \cap A}\left(\varepsilon m^{2}\right)^{-d_{i}-1 / 2}\left(v+\varepsilon m^{2}\right)^{-1 / 2\left|\Lambda \cap\left(\Sigma^{*} \backslash X^{*}\right)\right|} \\
& \left.\left(m^{2}+2 v\right)^{+\left|\Sigma^{*} \cup X^{*}\right|}\right\}\left\{\pi^{-(1 / 2)\left|\Sigma^{*} \cup X^{*}\right|} \prod_{i \in X^{*}} \Gamma\left(d_{i}+\frac{1}{2}\right) \pi^{(1 / 2)\left|\Sigma^{*}\right| X^{*} \mid} 2^{-\left|A \cap\left(\Sigma^{*} \cup X^{*}\right)\right|}\right. \\
& \left.2^{-(1 / 2)\left|\partial+\Lambda \cap\left(\Sigma^{*} \cup X^{*}\right)\right|} \cos \theta^{-2\left|\Sigma^{*} \cup X^{*}\right|-\Sigma d_{i}}\right\} . \\
& \text { This yields } \\
& \left|\tilde{\phi}_{\lambda, m}(\Sigma, X)\right| \leqq \exp \left(-k\left|\Sigma^{*} \cup X^{*}\right|\right) .
\end{aligned}
$$

where $k=k(m, \lambda, \theta)>0$ for $m^{2}$ large enough. This concludes the proof of Lemma 2 .

\section{The Algebraic Formalism. Proof of Theorem 1}

We will now briefly summarize the algebraic formalism which we need for the proof of Theorem 1, for details see Gallavotti et al. [4] and Bricmont et al. [1] for the pressure and Seiler [8], Constantinescu, Klöckner [2] for the correlation functions.

\section{a) The Pressure}

Recall that a polymer is defined as a connected component of $\Sigma \cup X$ and let $\mathscr{P}$ be the set of all such polymers in any $\Lambda \subset \mathbb{Z}^{v}$. The elements of $\mathscr{P}$ are called $p_{1}, p_{2}, \ldots$ Let $\mathscr{F}$ be the set of multiindices (multiplicity functions) on $\mathscr{P}$. For $p \in \mathscr{F}$ we define

$$
\phi(P)=\left\{\begin{array}{l}
0 \text { if } P(p)>1 \text { for some } P \\
\prod_{i=1}^{n} \tilde{\phi}\left(p_{i}\right) \prod_{i<j}\left(1+g\left(p_{i}, p_{j}\right)\right)
\end{array}\right.
$$


where $\tilde{\phi}\left(p_{i}\right)$ is defined by (3.5a). Calling $p_{1}, \ldots, p_{n}$ the elements of $P$ (or supp $P$ ) for which $P(p)=1$ we can define a compatibility function $g$ by

$$
g\left(p, p^{\prime}\right)=\left\{\begin{array}{l}
0 \text { if } p, p^{\prime} \text { are disconnected } \\
-1 \text { otherwise. }
\end{array}\right.
$$

This allows us to rewrite (3.5b), which is really the sum of our admissible partitions, as

$$
\tilde{Z}(\lambda, m)=\sum_{P \subset A} \phi(P) .
$$

Note that $P(p)=0$ if $p \not \subset \Lambda$. The $\phi(P)$ can be interpreted as the coefficients of a formal power series $\Sigma_{P} \phi(P) X^{P}$, where $X^{P}=\Pi_{p} X_{p}^{P(p)}$.

In (5.3) we have set $X_{p}=1$ for all $p$. The $\phi(P)$ can be interpreted as Boltzmann factors and the corresponding Ursell functions can be found by the formalism described by Gallavotti et al. [4].

We define $\phi^{T}(P)$ as the coefficients of the formal power series $\log \left(\Sigma_{P} \phi(P) X^{P}\right)$. Explicitly

$$
\phi^{T}(P)=\sum_{n=1}^{\infty} \frac{(-1)^{n}}{n} \Sigma^{\prime} \prod_{i=1}^{n} \phi\left(P_{i}\right)
$$

where the sum $\Sigma^{\prime}$ runs over all $P_{1}, \ldots, P_{n} \in \mathscr{F}$, such that $P_{i} \neq 0$ and $\sum_{i=1}^{n} P_{i}=P$, the addition being defined pointwise. Thus we have (formally at least)

$$
\tilde{Z}_{\Lambda}(\lambda, m)=\exp \left(\sum_{P \subset A} \phi^{T}(P)\right) .
$$

This expression is justified by Lemma 2. Gallavotti et al. [4] and Bricmont et al. [5] showed that for polymer systems with exponential decay of the Boltzmann factors a bound of the following type exists independent of $\Lambda$ : for any $i \in \mathbb{Z}^{v}$

$$
\sum_{i \in P^{*}}\left|\phi^{T}(P)\right|<\infty .
$$

Now we can easily deduce our result for the pressure. Using Lemma 2,(5.6) and the properties of the $g_{i}(\lambda, m)$ we have for any sector $\{\lambda|| \arg \lambda|\leqq \pi / 2-\delta,| \lambda \mid<\varepsilon\}$ with $\varepsilon$ small enough and $m$ large:

$$
\log Z_{\Lambda}(\lambda, m)=\sum_{i \in \Lambda} \log g_{i}(\lambda, m)+\sum_{P \subset \Lambda} \phi^{T}(P)
$$

It is evident that $|\Lambda|^{-1} \log Z_{\Lambda}(\lambda, m)$ is uniformly bounded on any compact subset of the above sector. Applying Vitali's theorem analyticity of the pressure in the infinite volume limit follows.

\section{b) The Correlation Functions}

We will now briefly sketch the method for obtaining analyticity of the correlation functions. We introduce modified partition functions following a method of $E$. Seiler [8]. For details see Constantinescu and Klöckner [2]. Let $A$ be a multiindex on $\mathbb{Z}^{v}$ with $A(\varphi)=\prod_{i \in \operatorname{supp} A} \varphi_{i}^{A(i)}$. For $\beta \in \mathbb{C}$ let $A(\beta)(\varphi)=1+\beta A(\varphi)$. 
Let

$$
Z_{A, A(\beta)}(\lambda, m)=\int_{\mathbb{R}^{|\bar{A}|}} A(\beta)(\varphi) \exp \left(\sum_{|i-j|=1} \varphi_{i} \varphi_{j}\right) \prod_{i \in \bar{A}} d \mu_{i}(\varphi)
$$

where

$$
d \mu_{i}(\varphi)=\left\{\begin{array}{l}
g_{i}(\lambda, m)^{-1} \exp \left(-v \varphi^{2}-P(\varphi)\right) d \varphi, i \in \Lambda \\
g_{i}(\lambda, m)^{-1} \exp \left(-v \varphi^{2}-\frac{m^{2}}{2}(\varphi-\xi)^{2}\right) d \varphi, i \in \partial_{+} \Lambda
\end{array}\right.
$$

We then have

$$
\left.\frac{d}{d \beta} \log Z_{A, A(\beta)}(\lambda, m)\right|_{\beta=0}=\langle A\rangle_{A}(\lambda) .
$$

We can now proceed with the contour cluster expansion as in Sect. 3. With the notations of Sect. 3 we have

$$
\begin{aligned}
& Z_{\Lambda, A(\beta)}(\lambda, m)=\sum_{\Sigma}\left[(-1)^{|\Lambda-(\Sigma)|} \exp \left(-\xi^{2}\left(v|\bar{\Lambda}|+|\Sigma|-\left|\Sigma^{c}\right|\right)\right)\right. \\
& \cdot \sum_{X \subset \Sigma^{c}} \int_{\mathbb{R}^{|\bar{\Lambda}|}}\left\{\left(1+\beta(-1)^{|\Lambda-(\Sigma) \cap \operatorname{supp} A|} A(\psi+\xi)\right) \prod_{i \in \Lambda} \chi_{+}\left(\psi_{i}+\xi\right) \prod_{(i j) \in \Sigma} \exp \left(-\psi_{i} \psi_{j}\right)\right. \\
& \cdot \prod_{(i j) \in X}\left(\exp \left(\psi_{i} \psi_{j}\right)-1\right) \exp \left(-\sum_{i \in \Sigma^{*} \cup \partial_{+} \Lambda} 2 c_{i} \xi \psi_{i}-\sum_{i \in \Lambda} Q\left(\psi_{i}\right)\right) \\
& \left.\left.\cdot \exp \left(-\frac{1}{2} m^{2} \sum_{i \in \partial+\Lambda} \psi_{i}^{2}-v \sum_{i \in \bar{\Lambda}} \psi_{i}^{2}\right)\right\} \prod_{i \in \bar{\Lambda}} g_{i}(\lambda, m)^{-1} d \psi_{i}\right]
\end{aligned}
$$

Instead of (5.10) we will study

$$
\tilde{Z}_{A, A(\beta)}(\lambda, m)=\langle A(\beta)\rangle_{0}^{-1} Z_{A, A(\beta)}(\lambda, m),
$$

where

$$
\langle A(\beta)\rangle_{0}=\int_{\mathbb{R}^{|\overline{\mid}|}} A(\beta)(\varphi) \prod_{i \in \bar{\Lambda}} d \mu_{i}(\varphi)
$$

Let us call $p$ a polymer, if it is either a connected component of $\Sigma \cup X$ not intersecting supp $A$ or the (disjoint) union of all connected components intersecting supp $A$. Thus a compatibility function can be defined for such polymers by

$$
g_{A}\left(p, p^{\prime}\right)=\left\{\begin{array}{l}
0 \text { if the supports are disjoint and not both } p \text { and } p^{\prime} \text { intersect supp } A \\
-1 \text { otherwise. }
\end{array}\right.
$$

The integrals in (5.10) factor over such polymers. Let us define

$$
n(p)=\left\{\begin{array}{l}
1 \text { if } p^{*} \cap \operatorname{supp} A \neq \varnothing \\
0 \text { otherwise }
\end{array}\right.
$$


and

$$
\tilde{p}^{*}=\left\{\begin{array}{l}
p^{*} \text { if } p^{*} \cap \operatorname{supp} A=\varnothing \\
p^{*} \cup \operatorname{supp} A \text { if } p^{*} \cap \operatorname{supp} A \neq \varnothing .
\end{array}\right.
$$

We can then rewrite (5.11a) in the same way as (5.3)

$$
\tilde{Z}_{\Lambda, A(\beta)}(\lambda, m)=\sum_{P \subset A} \phi_{A(\beta)}(P) .
$$

where $P$ is a multiindex and $P \subset \Lambda$ means $p \subset \Lambda$ for all $p$ with $P(p)=1$. We have the Boltzmann factors

$$
\phi_{A(\beta)}(P)=\left\{\begin{array}{l}
\tilde{\phi}_{A(\beta)}(p) \text { if } P=(p) \\
\prod_{i=1}^{n} \tilde{\phi}_{A(\beta)}\left(p_{i}\right) \prod_{i \leqq j}\left(1+g_{A}\left(p_{i}, p_{j}\right)\right),
\end{array}\right.
$$

where

$$
\begin{aligned}
& \tilde{\phi}_{A(\beta)}(p)=(-1)^{\left|\Lambda-(\Sigma) \cap \tilde{p}^{*}\right|} \exp \left(-\xi^{2}\left(v\left|\bar{\Lambda} \cap p^{*}\right|+\left|\Sigma \cap p^{*}\right|-\left|\Sigma^{c} \cap p^{*}\right|\right)\right) \\
& \langle A(\beta)\rangle_{0}^{-n(p)} \int_{\mathbb{R}^{\mid} \tilde{p} * \mid}\left(1+\beta(-1)^{|\Lambda-(\Sigma) \cap \operatorname{supp} A|} A(\psi+\xi)\right)^{n(p)} \prod_{i \in \Lambda \cap \tilde{p}^{*}} \chi_{+}\left(\psi_{i}+\xi\right) \\
& \prod_{(i j) \in \Sigma \cap p} \exp \left(-\psi_{i} \psi_{j}\right) \prod_{(i j) \in X \cap p}\left(\exp \left(\psi_{i} \psi_{j}\right)-1\right) \\
& \quad \exp \left(-\sum_{i \in\left(\Sigma^{*} \cup \partial_{+} \Lambda\right) \cap \tilde{p}^{*}} 2 c_{i} \xi \psi_{i}-\sum_{i \in A \cap \tilde{p}^{*}} Q\left(\psi_{i}\right)\right) \\
& \exp \left(-\frac{1}{2} m^{2} \sum_{i \in \partial+\Lambda \cap \tilde{p}^{*}} \psi_{i}^{2}-v \sum_{i \in \bar{A} \cap \tilde{p}^{*}} \psi_{i}^{2}\right) \prod_{i \in \tilde{p}^{*}} g_{i}(\lambda, m)^{-1} d \psi_{i} .
\end{aligned}
$$

We can now apply our algebraic formalism and obtain

$$
\log \tilde{Z}_{\Lambda, A(\beta)}(\lambda, m)=\sum_{P \subset A} \phi_{A(\beta)}^{T}(P)
$$

with $\phi_{A(\beta)}^{P}(P)$ defined as in (5.2). Taking into account (5.4) we notice that differentiating with respect to $\beta$ reduces to differentiating $\tilde{\phi}_{A(\beta)}(p)$, where supp $A \cap_{p} \neq \varnothing$. Let $\phi_{A}(p)$ be defined by $(5.13 b)$ with $A(\beta)$ and $\left(1+\beta(-1)^{|A(\Sigma) \cap \operatorname{supp} A|}(A(\psi+\xi))\right.$ replaced by $A$ and $(1-)^{|\Lambda-(\Sigma) \cap \operatorname{supp} \Lambda|} A(\psi+\xi)$ respectively and let $\langle A\rangle_{0}$ be defined in a similar fashion.

Then

$$
\left.\frac{d}{d \beta} \tilde{\phi}_{A(\beta)}(p)\right|_{\beta=0}=\langle A\rangle_{0}\left(\tilde{\phi}_{A}(p)-\tilde{\phi}(p)\right) .
$$

Note that

$$
\langle A\rangle_{\Lambda}(\lambda)=\langle A\rangle_{0}+\left.\sum_{\substack{\operatorname{supp} A \cap P \neq \varnothing \\ P \subset A}} \frac{d}{d \beta} \phi_{A(\beta)}^{T}(p)\right|_{\beta=0} .
$$

As we can bound (5.15) exponentially in the fashion of Sect. 4, we can employ the 
same reasoning as for the pressure, obtaining an estimate analogous to (5.6). For details see Constantinescu and Klöckner [2]. Vitali's theorem gives us the desired result and the theorem is proved.

\section{Appendix}

In this appendix we will prove (3.4e). Here $g_{i}(\lambda, m)$ does not depend on $i$ for $i \in \Lambda$, therefore we will drop the subscript. In the following we will set $|\arg \lambda| \leqq \pi / 2-\delta$. We have

$$
g(\lambda, m)=\int_{-\infty}^{\infty} \exp \left(-p(\varphi)-v(\varphi-\xi)^{2}\right) d \varphi-\int_{-\infty}^{\infty} \chi_{-}(\varphi) \exp \left(-p(\varphi)-v(\varphi-\xi)^{2}\right) d \varphi .
$$

The first part of the right-hand side will be called $\hat{g}(\lambda, m)$. The second part can be further decomposed. After scaling $\varphi$ we obtain

$$
\begin{aligned}
& \int_{-\infty}^{\infty} \chi_{-}(\varphi) \exp \left(-p(\varphi)-v(\varphi-\xi)^{2}\right) d \varphi=\lambda^{-1 / 2} \\
& \int_{0}^{\infty} \chi_{-}\left(\frac{\varphi}{\sqrt{\lambda}}\right) \exp \left(-\frac{1}{\lambda}\left(\left(\varphi^{2}-\frac{m^{2}}{8}\right)^{2}+v\left(\varphi-\frac{m}{\sqrt{8}}\right)^{2}\right) d \varphi-\lambda^{-1 / 2}\right. \\
& \int_{0}^{\infty} \chi_{+}\left(\frac{\varphi}{\sqrt{\lambda}}\right) \exp \left(-\frac{1}{\lambda}\left(\left(\varphi^{2}-\frac{m^{2}}{8}\right)^{2}+v\left(\varphi+\frac{m}{\sqrt{8}}\right)^{2}\right)\right) d \varphi .
\end{aligned}
$$

The second integral can easily be bounded by $|\lambda|^{-1 / 2} \lambda^{1 / 2} e^{-1 / \lambda^{\prime} v\left(m^{2} / 8\right)}$, where $\lambda^{\prime}=\left(\operatorname{Re} \lambda^{-1}\right)^{-1}=|\lambda| \cos \theta^{-1}, \theta=\arg \lambda$.

For the first integral note that

$$
\left|\chi_{-}\left(\frac{\varphi}{\sqrt{\lambda}}\right)\right| \leqq \pi^{-1 / 2}(1-\alpha)^{-1 / 2} \cos \theta^{-1 / 2} e^{-\left(\alpha / \lambda^{\prime}\right) \varphi^{2}} \text { for } \varphi \geqq 0 \text { and } \alpha \in[0,1),
$$

cf. Constantinescu, Ströter [3] or Glimm, Jaffe, Spencer [5].

This allows us to bound this integral by

$$
\begin{gathered}
|\lambda|^{-1} \pi^{-1 / 2}(1-\alpha)^{-1 / 2} \int_{0}^{\infty} \exp \left(-\frac{1}{\lambda^{\prime}}\left(\left(\varphi^{2}-\frac{m^{2}}{8}\right)^{2}+v\left(\varphi-\frac{m}{\sqrt{8}}\right)^{2}+\alpha \varphi^{2}\right)\right) \mathrm{d} \varphi . \\
\left(\varphi^{2}-\frac{m^{2}}{8}\right)^{2}+v\left(\varphi-\frac{m}{\sqrt{8}}\right)^{2}+\frac{\alpha}{2} \varphi^{2} \geqq \text { const } m^{2}>0 \text {, so that this can again be }
\end{gathered}
$$
bounded by $\cos \theta^{-1} \exp \left(-\operatorname{const}\left(m^{2} / \lambda^{\prime}\right)\left(\pi^{-1 / 2} / 2\right)(1-\alpha)^{-1 / 2} \alpha^{-1 / 2}\right.$.

Thus we see that the second part of the right-hand side of (A1) decays exponentially fast for $\lambda \rightarrow 0$ (or $\xi \rightarrow \infty$ ).

Let us now consider $\hat{g}(\lambda, m)$. We have

$$
\hat{g}(\lambda, m)=\int_{0}^{\infty} \exp \left(-p(\varphi)-v(\varphi-\xi)^{2}\right) d \varphi+\int_{0}^{\infty} \exp \left(-p(\varphi)-v(\varphi+\xi)^{2}\right) d \varphi .
$$

The second integral decays exponentially for $\lambda \rightarrow 0$. This is immediately clear from 
the above reasoning. It remains to study the asymptotic properties of

$$
\tilde{g}(\lambda, m)=\int_{0}^{\infty} \exp \left(-p(\varphi)-v(\varphi-\xi)^{2}\right) d \varphi .
$$

Scaling $\varphi$ as above we obtain

$$
\tilde{g}(\lambda, m)=\lambda^{-1 / 2} \int_{0}^{\infty} \exp \left(-\frac{1}{\lambda} f(\varphi)\right) d \varphi .
$$

Here $f(\varphi)=\left(\varphi^{2}-\left(m^{2} / 8\right)\right)^{2}+v(\varphi-(m / \sqrt{8}))^{2}$ attains a minimum 0 at $\varphi_{0}=m / \sqrt{8}$ with $f^{\prime \prime}\left(\varphi_{0}\right) \neq 0$. We now apply the complex Laplace method (see Olver [7], p. 125ff) and we get

$$
\tilde{g}(\lambda, m) \sim 2 \sqrt{\pi}\left(m^{2}-2 v\right)^{-1 / 2}+0\left(\frac{1}{\lambda}\right)
$$

uniformly in any sector $|\arg \lambda| \leqq \frac{1}{2} \pi-\delta \Lambda \delta>0$.

It was not possible to construct a path of steepest descent for an integral with $|\arg \lambda| \geqq \pi / 2$ and rotated contours. This seems to be another indication that Borel summability cannot be obtained.

Acknowledgements. The authors are indebted to Prof. F. Constantinescu, Frankfurt, for valuable suggestions and discussions.

\section{References}

1. Bricmont, J., Lebowitz, J. L., Pfister, C. E.: Low temperature expansion for continuous spin Isingmodels. Commun. Math. Phys. 78, 117-135 (1980)

2. Constantinescu, F., Klöckner, K.: Absence of phase transitions and analyticity in the physical parameters. (to appear)

3. Constantinescu, F., Ströter, B.: The Ising limit of the double well model.J. Math. Phys. 21, 881-891 (1980)

4. Gallavotti, G., Martin-Löf, A., Miracle-Sole, S.: Some problems connected with the description of coexisting phases at low temperatures in the Ising model. In: Statistical mechanics and mathematical problems. Batelle-Rencontres 1971. Lecture Notes in Physics, Vol. 20. Berlin, Heidelberg, New York: Springer 1973

5. Glimm, J., Jaffe, A., Spencer, T.: A convergent expansion about mean field theory, I, II. Ann. Phys. 101, $610-630,631-669$ (1976)

6. Nevanlinna, F.: Zur Theorie der asymptotischen Potenzreihen. Ann. Acad. Sci. Fenn. Ser. A12,(1918 1919)

7. Olver, F. W. J. : Asymptotics and special functions. New York, San Francisco, London: Academic Press 1974

8. Seiler, E.: Geneva Lecture Notes 1981. (private communication)

9. Sokal, A. D.: An improvement of Watson's theorem on Borel summability. J. Math. Phys. 21, 261-263 (1980)

Communicated by A. Jaffe

Received March 22, 1982; in revised form May 10, 1982 